\title{
ANÁLISIS DE LOS TRABAJOS PRÁCTICOS DE QUÍMICA GENERAL EN UN PRIMER CURSO DE UNIVERSIDAD
}

\author{
INSAUSTI, M.J. \\ Departamento de Química-Física. Facultad de Ciencias. Universidad de Valladolid. \\ 47005 Valladolid.
}

\section{SUMMARY}

Learning in the laboratory has been and still is a highly controversial issue among science educators although there are not many studies carried out in Spanish Universities. This paper reports an evaluation of the current situation in a Laboratory of General Chemistry.

For the purpose of this research, a model of learning has been adopted and an evaluation of students attitude towards the Practical Works has been made.

\section{INTRODUCCIÓN}

En la bibliografía aparecen con frecuencia trabajos críticos y tambien propuestas de renovación para la tarea de laboratorio. Se indica que los estudiantes no tienen ideas claras de lo que están haciendo, no son capaces de relacionar los conceptos y fenómenos involucrados en el experimento, y además no ven la experimentación como un proceso de construccion del conocimiento (Woolnough y Allsop, 1985). Otras críticas nos indican que los trabajos prácticos (TP) no reflejan las características esenciales del trabajo científico y, por tanto, no contribuyen a que los alumnos se familiaricen con la metodologia científica. Plantean la hipotesis de que «es posible transformar los TP de forma que posean las características de un trabajo de investigación y conduzcan a una efectiva familiarización de los alumnos con los aspectos esenciales de la metodología científica, al tiempo que contribuyen a la (re)construcción de conocimientos, superando la simple transmisión-recepción de conocimientos ya elaborados» (Payá, 1991). Hodson (1994) nos indica que muchas de las dificultades discutidas con anterioridad son debidas a la manera irreflexiva en que las personas encargadas de los TP hacen uso del laboratorio.

Estudios realizados sobre los TP de química en universidades inglesas hablan de los malos resultados en cuanto a la construccion de conocimiento científico, adquisición de destrezas, actitudes positivas de los alumnos, etc. (Johnstone y Letton, 1990; Maester y Maskill, 1993). Existen muchos estudios y propuestas de renovación de los trabajos prácticos realizados en otros países que contrastan con los escasos estudios realizados en las universidades españolas en el campo de la química.

Creo que es importante antes de realizar cambios, estudiar cómo están encuadradas nuestras prácticas y lo que piensan nuestros estudiantes sobre los TP que están realizando. Éste es el objetivo del presente trabajo sobre las prácticas de química en el primer curso de la carrera de Ciencias Químicas. Intentamos colaborar en hacer 
existe una mayor dispersión de las respuestas, por lo que presentamos los resultados como porcentaje de aspecto positivo, negativo y neutro. Analizaremos los resultados que nos conducen hacia el objetivo de detectar los principales problemas con los que se enfrentan los estudiantes en un laboratorio. Los resultados más en desacuerdo, que serán los que analizaremos aquí (Tabla I), son los relacionados con la dificultad conceptual que involucra la práctica (ítems $2,4,11$ y 12). Los demás items que inciden sobre los objetivos organización de laboratorio y organización del manual son evaluados positivamente por los alumnos.

Analizamos los resultados de cuatro TP: velocidad de reacción (VR), ley de Hess (LH), volumetría ácido fuerte-base fuerte (AB) y destilación de un vino (DV). La realización de las dos primeras prácticas involucran cálculos que implican más conceptos, al contrario de las dos últimas, las cuales, por otra parte, ya habían sido realizadas por un porcentaje alto de alumnos en cursos anteriores. Los resultados son mucho más negativos para las dos primeras prácticas que para las dos últimas.

Los resultados positivos referentes a su visión de los manuales y situación de laboratorio, así como los ftems $2,7,8,9,11$ de la figura 1 , contrastan con los estudios realizados en otras universidades. Quizás el carácter predominantemente teórico de los estudios realizados en la enseñanza secundaria, con poco trabajo experimental, explicaría la buena valoración hecha por parte de nuestros estudiantes.

\section{CONCLUSIONES}

Como se indicó al principio de este artículo, el propósito de este estudio es explorar la actitud de los estudiantes hacia los TP y detectar los problemas que tienen frente a un laboratorio de química general. Nuestros resultados principales ya han sido mostrados aquí y los resumiremos en los siguientes apartados:

1. Los alumnos tienen una actitud positiva respecto al laboratorio realizado en cursos de bachilierato y el que realizan en $1^{\circ}$ de universidad, en cuanto a aspecto lúdico y necesario de las prácticas.

2. Su actitud no es tan positiva y en algunos casos es negativa en cuanto a la organización del laboratorio y material presentado.

3. La dificultad para nuestro alumno está en el contenido conceptual y técnicas experimentales involucradas en las prácticas. Podemos decir que éste es el "ruido" detectado. Este apartado viene además avalado por lo que está reflejado en el diarjo del profesor de prácticas, que confecciona a partir de las preguntas de los alumnos. También nos encontramos con gran cantidad de errores en sus informes finales de los TP.

4. La crítica generalizada en estudios educativos de TP sobre que los alumnos siguen los manuales como «recetas de cocinan, es otra conclusión a la que podemos llegar, ya que, como ellos mismos nos indican, muy

\begin{tabular}{|l|c|c|c|c|}
\hline Ítem & Práctica & \% A y B & $\%$ C & $\%$ D y E \\
\hline 2. He tenido suffciente tiempo en el laboratorio para & VR & 33 & 20 & 47 \\
pensar en los conceptos químicos involucrados en la & LH & 35 & 18 & 47 \\
experiencia. & AB & 55 & 16 & 29 \\
& DV & 80 & 0 & 20 \\
\hline 4. Me hubiera gustado haber tenido más ayuda para & VR & 63 & 27 & 10 \\
hacer los cátculos involucrados en el experimento. & LH & 72 & 0 & 19 \\
& AB & 35 & 16 & 49 \\
& DV & 30 & 10 & 60 \\
\hline 11. Conozco bien las técnicas experimentales, por lo & VR & 20 & 30 & 50 \\
que he podido estar concentrado en los conceptos & LH & 30 & 20 & 50 \\
químicos involucrados en la experiencia. & AB & 59 & 19 & 22 \\
& DV & 70 & 15 & 15 \\
\hline 12. Podría repetir esta experiencia en cualquier & VR & 40 & 10 & 50 \\
momento. & LH & 41 & 11 & 48 \\
& AB & 93 & 3 & 4 \\
& DV & 90 & 7 & 3 \\
\hline
\end{tabular}


Figura 2

Frecuencia de respuestas de los alumnos a los precuestionarios y postcuestionarios.

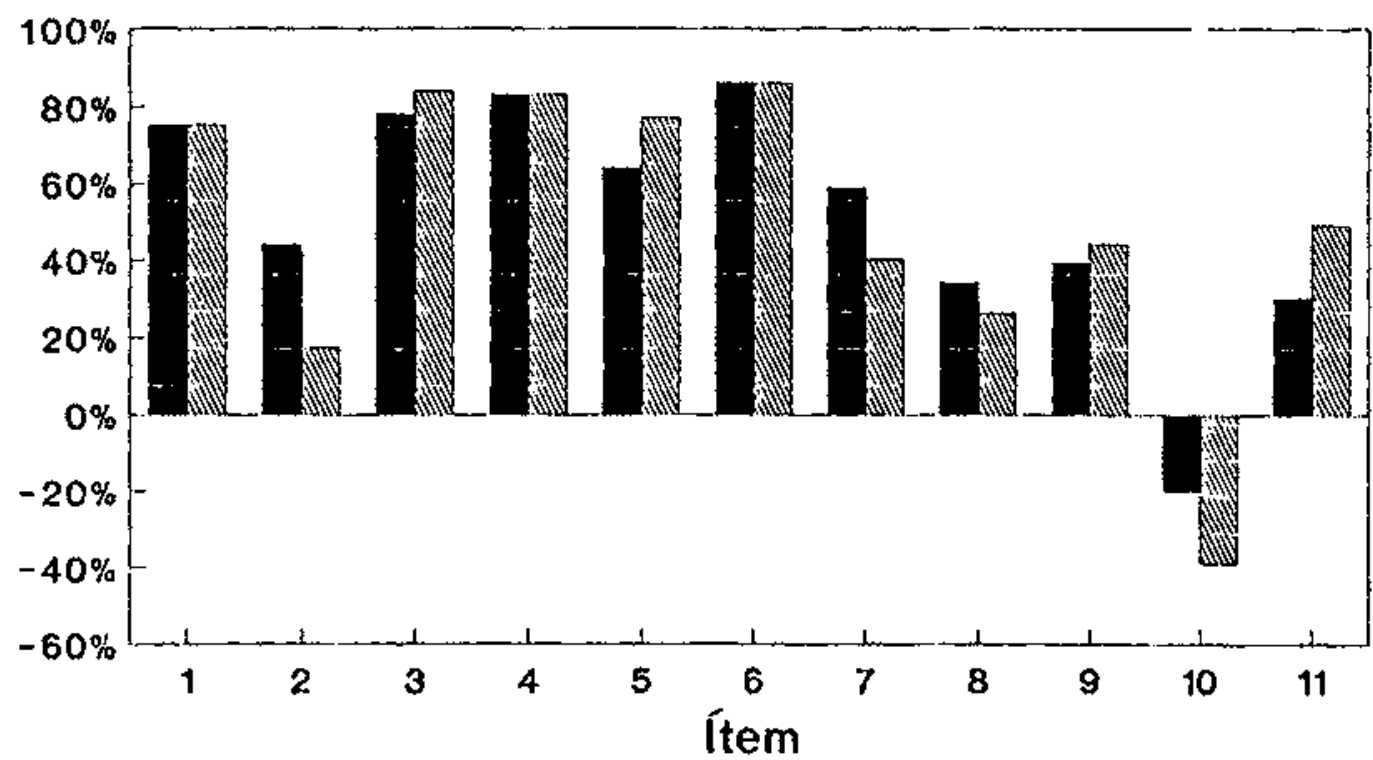

precuestionario postcuestionario

pocos se encuentran en situación de poder repetir determinadas prácticas.

Para evitar en los alumnos la situación de sobrecarga que se puede crear en la realización de las prócticas, debemos emprender la tarea de cambiar la forma de trabajar en el laboratorio. Johnstone y Letton (1990 y 1991) así como Lynch (1987) nos hacen sugerencias de los cambios que se deben realizar en un laboratorio de química de los primeros cursos de universidad. Estos cambios nos llevarían a la introducción de un prelaboratorio y de un postlaboratorio, así como una modificación de los manuales de prácticas.

En el prelaboratorio el estudiante pondría a punto los cálculos y técnicas instrumentales de los TP, es decir organizaría su pensamiento antes de entrar al laboratorio. No se les permitiráa empezar la práctica experimental propiamente dicha sin que el profesor revise esta parte.

En el postlaboratorio (una vez realizada la práctica experimental) se llevarían a cabo las interpretaciones y discusiones sobre el trabajo realizado. Es en este apartado donde también se introducirían pequeñas investigaciones que tienen que ser resultas por medios prácticos, usando técnicas y contenidos conceptuales ya involucra. dos en las prácticas anteriormente realizadas. Estos problemas prácticos alentarían al estudiante al trabajar independientemente, planificar su trabajo y obtener sus propias conclusiones.

Como queda reflejado en el inicio de este trabajo, creo que los cambios que se vayan a introducir deben ser analizados y comparados con los actualmente obtenidos.

\section{AGRADECIMIENTOS}

Deseo agradecer al Dr. Alex Jonhstone (Universidad de Glasgow) el tiempo que me ha dedicado para discutir sobre los trabajos prácticos. 


\section{REFERENCIAS BIBLIOGRÁFICAS}

BADDELEY, A. (1986). Working Memory. Oxford University press.

BOUND, D., DUNN J., y HEGARTY-HAZEL, E. (1989). Teaching in Laboratories. Open University Press.

COHEN, L. y MANION, L. (1990). Métodos de investigación educativa. Madrid: La Muralla.

ISCUDERO ESCORZA, T. (1995). La evaluación de las actitudes cientificas Alambique, 4, pp. 33-41.

ESPINOSA GARCÍA, J. y ROMÁN GALÁN, T. (1993). Actitudes hacia la ciencia en estudiantes universitarios de ciencias. Enseñanza de las Ciencias, 11(3), pp. 297-300.

GAGNE, R.M. (1987). Lascondiciones del aprendizaje. México, D.F: 'Trillas.

GIL.PÉREZ, D. (1994). Diez años de investigación en didactica de las ciencias: realizaciones y perspectivas. Enseñanza de las Ciencias, 12(2), pp. 154-158.

HERRON, M.D. (1971). The nature of scientific inquiry. School Rewiew, 79, pp. 141-212.

HODSON, D. (1994). Hacia un enfoque más crítico del trabajo de laboratorio. Enseñanza de las Ciencias, 12(3), pp. 299-313.

JOHNSTONE, A.H., y WHAM, J.B. (1982). The demand of practical work. Education in Chemistry, 19, pp. 71-74.
JOHNSTONE, A.H, y I.E'TON, K.M. (1990). Investigating undergraduate laboratory work. Education in Chemistry, I, pp. 9-1I;

JOHNSTONE, A.H. y LETTON K.M. (1990). Practical measures for practical work. Education in Chemistry, 5, pp. 81-83.

JOHNSTONE, A.H. y LETTON K.M. (1991). Why dopractical work? A researcher's point of view. International Newsletter on Chemical Education, 34, pp. 14-18.

KEMPA, R. F., y NICHOLLS, C.E. (1983). Problem-solving ability and cognitive structure: an exploratory investigation, European Journal of Science Education, 5, pp. 171-184.

LYNCH, R.P. (1987). Laboratory work in schools and universities: structures and strategies still largely unexplored. The Atstralian Science Teachers Journal, 32(4), pp. 31-39.

MAESTER, A.M., y MASKILL, R. (1993). First year practical classes in undergraduate chemistry courses in England and Gales. Education in Chemistry, 11, pp. 156-159.

PAYÀ, J. (1991). Tesis doctoral, Universidad de Valencia.

PICKERING, M. y MONTS, L. D. (1982). How students reconcile discordant data. Journal of Chemical Education, 59(9), pp. 794-796.

SANFORD, A.J. (1985). Cognition and Cognitive Psychology. Londres: Weidenfeld and Nicloson.

WOOLNOUGH, B, y AlLSSOP, T. (1985). Practical Work in Science. Cambridge Science Fducation Series. 


\section{ANEXO I}

Se está intentando llevar a cábo un trabajo sobre el laboratorio. Nos gustaría conocer tu experiencia previa antes de este curso (precuestionario) sobre las prácticas realizadas el presente curso (postcuestionario).

Tu verdadera opinión nos será muy valiosa y será tratada con estricta confidencia.

De acuerdo con tu opinión, rellena la tabla siguiente, después de fijarte en el ejemplo.

Si encuentras que el laboratorio ha sido muy aburrido, debes poner una cruz como sigue:
interesante
0000
aburrido

Si crees que fue algo interesante, debes poner la cruz en:
interesante
0000
aburrido

Tabla:

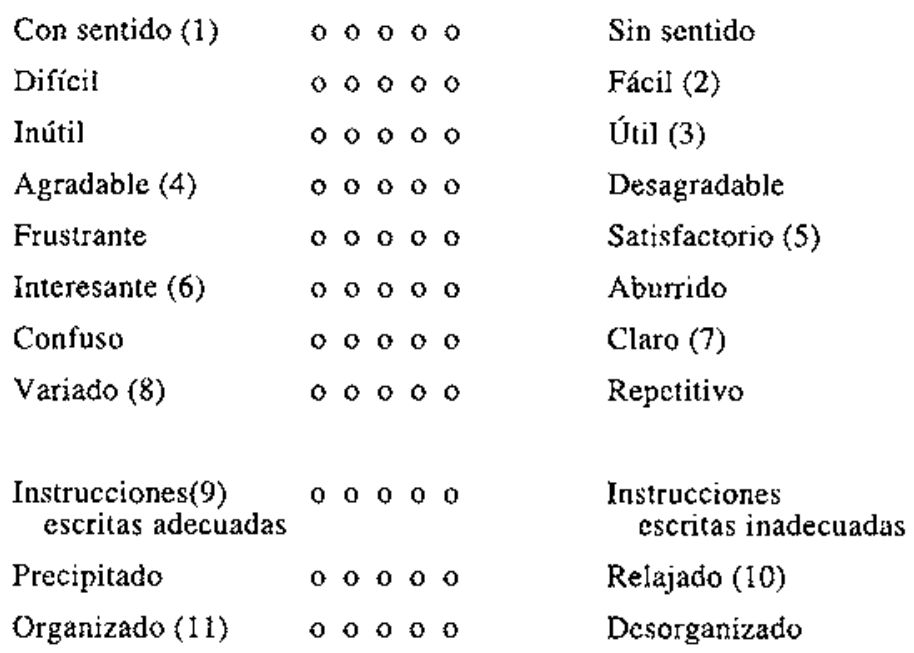

NOTA: Los números entre paréntesis corresponden a los ítems de la figura 1. 


\section{ANEXO II}

Por favor, contesta según tu experiencia en la práctica que estés realizando. Indica el grado de acuerdo o desacuerơo con las siguientes sentencias, marcando una cruz en el ítem apropiado.

Tus indicaciones serán tratảas con estricta confidencia y no afectarán en absoluto tu evaluación en el laboratorio.

1. Hay suficiente información en el laboratorio y en el manual para ayudarme a encontrar los productos químicos.

2. He tenido suficiente tiempo en el laboratorio para pensar los conceptos químicos involucrados en la experiencia.

3. Está claro para mí, qué se espera que entregue en cl informe al finalizar las prácticas.

4. Me hubiera gustado haber tenido más ayuda para hacer los cálculos involucrados en el experimento.

5. Fn el manual estaban suficientemente explicadas las técnicas para realizar la experiencia.

6. Fi procedimiento experimental está claramente explicado en el manual.

7. Fs muy fácil seguir el manual, está muy bien organizado (objetivos, seguridad, precauciones, procedimiento experimental).

8. Es muy confuso este manual y elto me ha llevado a seguir las instrucciones sin tealmente saber lo que hago.

9. E] objetivo del experimento está claro para mí,

10. El trabajo experimental es una parte básica del curso.

11. Conozco muy bien las técnicas experimentales, por lo que he podido estar concentrado en los conceptos químicos involucrados en la experiencia.

12. Podría repetir la experiencia en cualquier momento.

13. Siento que la sesión ha sido positiva.
A: Muy de acuerdo
B: De acuerdo
C: Netro
D: En desacuerdo
E: Muy en desacuerdo 